\title{
Periodic Orbits in Rotating Second Degree and Order Gravity Fields
}

\author{
Wei-Duo $\mathrm{Hu}^{1}$ and Daniel J. Scheeres ${ }^{2}$ \\ 1 School of Astronautics, BeiHang University, Beijing 100083; weiduo.hu@ gmail.com \\ 2 Department of Aerospace Engineering, The University of Michigan, Ann Arbor, MI 48109, USA
}

Received 2007 May 8; accepted 2007 October 12

\begin{abstract}
Periodic orbits in an arbitrary 2nd degree and order uniformly rotating gravity field are studied. We investigate the four equilibrium points in this gravity field. We see that close relation exists between the stability of these equilibria and the existence and stability of their nearby periodic orbits. We check the periodic orbits with non-zero periods. In our searching procedure for these periodic orbits, we remove the two unity eigenvalues from the state transition matrix to find a robust, non-singular linear map to solve for the periodic orbits. The algorithm converges well, especially for stable periodic orbits. Using the searching procedure, which is relatively automatic, we find five basic families of periodic orbits in the rotating second degree and order gravity field for planar motion, and discuss their existence and stability at different central body rotation rates.
\end{abstract}

Key words: celestial / orbital mechanics — asteroid — periodic orbit — resonance

\section{INTRODUCTION}

Periodic orbits in the three body problem have now been studied in both theory and application for several decades (Szebehely 1967; Richardson 1980; Kulkarni 2006). Periodic orbits around asteroids begin to be investigated more recently. The study of periodic orbits around an irregularly shaped, uniformly rotating asteroid can help us understand the nature of orbital dynamics around asteroids in general. Practically, the study gives insight into conditions of general stability in planned orbital motion. Stable periodic orbits can be directly used for missions and even unstable periodic orbits, if stabilized, can be used to realize safe close proximity orbital missions. Scheeres et al. $(1996,2000)$ calculated and analyzed some families of periodic orbits for a number of specific asteroids. This paper studies periodic orbits around asteroids in general by modeling the asteroid perturbation potential with the most significant gravity coefficients, $C_{20}$ and $C_{22}$ (see Hu \& Scheeres 2002, 2004). Stability analysis of these orbits is performed using Floquet's theorem. A previous version was presented at the Ninth International Space Conference of Pacific-basin Societies, 2001, Pasadena, California.

First, we analyze the four equilibrium points in the rotating 2 nd degree and order gravity field. Two of these are always unstable, the other two can be stable or unstable, depending on the rotation rate of the central body, $\omega_{T}$, the two gravity coefficients, $C_{20}$ and $C_{22}$, and its mass (Hu \& Scheeres 2004). We find that the stability of these equilibria has close relation with the existence and stability of their nearby periodic orbits.

Next, we compute families of periodic orbits. In our searching procedure, we remove the two unity eigenvalues from the state transition matrix (STM) to find a robust, non-singular linear map to solve for the periodic orbits. The algorithm converges well, especially for stable periodic orbits. As we give the initial variations a Jacobi constraint, the procedure automatically searches for a periodic orbit at a fixed energy level.

By calculating the STM for the periodic orbit, we find the characteristic multipliers which are an index of stability of the periodic orbit. These are used to test the stability of the periodic orbit. 
Then, by using an essentially automatic searching procedure for periodic orbits, we find five basic families of periodic orbits and discuss their existence and stability at different central body rotation rates. We call these five basic families, near direct, far direct, 1:1 resonant, 1:2 resonant and retrograde periodic orbits. There are also other "special" periodic orbits which lie between these basic periodic orbit families.

\section{SPACECRAFT MODEL}

There are thousands of asteroids in our solar system, most of them are irregular in shape and with different rotating rates (Perko 2000). The coupling of the mass distribution of a distended asteroid and its rotation makes the orbit dynamics fundamentally different from that of slightly elliptical bodies, such as the Earth and other major planets in the solar system.

For our basic model we assume an attracting body with gravitational parameter $\mu$ and a 2 nd degree and order gravity field. We assume that the body-fixed coordinate axes are aligned along the body's principal moments of inertia along axes $x, y$ and $z$ ordered by $I_{x x} \leq I_{y y} \leq I_{z z}$. With this orientation the 2nd degree and order gravity field can be specified by two coefficients, $C_{20} \leq 0$ and $C_{22} \geq 0$. The relations between the gravity coefficients and the principal moments of inertia of the body (normalized by the body mass) are: $C_{20}=-1 / 2\left(2 I_{z z}-I_{x x}-I_{y y}\right), C_{22}=1 / 4\left(I_{y y}-I_{x x}\right)$. Finally, we assume that the asteroid is in uniform rotation about its maximum moment of inertia (the $z$-axis) with a rotation rate $\omega_{T}$ and a corresponding rotation period $T=2 \pi / \omega_{T}$.

Many methods have been used for the orbital motion about an oblate planet (or gravity field with $C_{20}$ term only). Most studies that have included the equatorial ellipticity of the central body (i.e., the $C_{22}$ gravity term) have made the assumption that the ellipticity is small, as is appropriate for planets in the solar system. Our current problem is a significant departure, however, in that it considers values of the $C_{22}$ gravity coefficient that are significantly larger than those found for all solar system planets, making this analysis applicable to asteroids and comets which can have large values of equatorial ellipticity and different rotation rates.

\subsection{Equations of Motion}

The motion of a spacecraft in the body-fixed, rotating frame is defined as the solution to the equations of motion:

$$
\begin{aligned}
\ddot{x}-2 \omega_{T} \dot{y} & =\omega_{T}^{2} x-\frac{\mu}{r^{3}} x+\frac{\partial U_{2}}{\partial x}, \\
\ddot{y}+2 \omega_{T} \dot{x} & =\omega_{T}^{2} y-\frac{\mu}{r^{3}} y+\frac{\partial U_{2}}{\partial y}, \\
\ddot{z} & =-\frac{\mu}{r^{3}} z+\frac{\partial U_{2}}{\partial z},
\end{aligned}
$$

where $r=\sqrt{x^{2}+y^{2}+z^{2}}$. The gravitational force potential of 2 nd degree and order in the body-fixed frame is

$$
U_{2}=\frac{\mu}{r^{3}}\left[C_{20}\left(1-\frac{3}{2} \cos ^{2} \delta\right)+3 C_{22} \cos ^{2} \delta \cos 2 \lambda\right],
$$

where $\delta$ is the particle's latitude and $\lambda$ is its longitude. It can be easily shown that the above system can

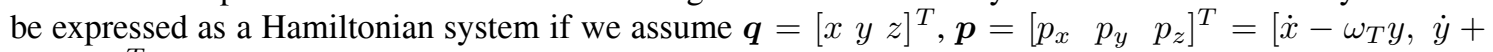
$\left.\omega_{T} x, \dot{z}\right]^{T}$.

\subsection{Gravity Potential and its Derivatives}

To calculate the state transition matrix, we need to know the second order partial derivatives of the gravity potential. The effective potential function in Cartesian coordinates is

$$
U=\frac{1}{2} \omega_{T}^{2}\left(x^{2}+y^{2}\right)+\frac{\mu}{r}-\frac{\mu C_{20}\left(x^{2}+y^{2}-2 z^{2}\right)}{2 r^{5}}+\frac{3 \mu C_{22}\left(x^{2}-y^{2}\right)}{r^{5}} .
$$


The first order partial derivatives of the potential are

$$
\begin{aligned}
U_{x}= & \omega_{T}^{2} x-\frac{\mu x}{r^{3}}-\frac{\mu C_{20} x}{r^{5}}+\frac{5 \mu C_{20} x\left(x^{2}+y^{2}-2 z^{2}\right)}{2 r^{7}} \\
& +\frac{6 \mu C_{22} x}{r^{5}}-\frac{15 \mu C_{22} x\left(x^{2}-y^{2}\right)}{r^{7}} \ldots
\end{aligned}
$$

The second order partial derivatives of the potential are

$$
\begin{aligned}
U_{x x}= & \omega_{T}^{2}-\frac{\mu}{r^{3}}+\frac{3 \mu x^{2}}{r^{5}} \\
& -\frac{\mu C_{20}}{r^{5}}+\frac{5 \mu C_{20}\left(5 x^{2}+y^{2}-2 z^{2}\right)}{2 r^{7}}-\frac{35 \mu C_{20} x^{2}\left(x^{2}+y^{2}-2 z^{2}\right)}{2 r^{9}} \\
& +\frac{6 \mu C_{22}}{r^{5}}-\frac{15 \mu C_{22}\left(5 x^{2}-y^{2}\right)}{r^{7}}+\frac{105 \mu C_{22} x^{2}\left(x^{2}-y^{2}\right)}{r^{9}} \ldots
\end{aligned}
$$

\subsection{State Transition Matrix}

Assume the system

$$
\dot{\boldsymbol{x}}=\boldsymbol{f}(\boldsymbol{x}) \quad \text { for } \quad \boldsymbol{x} \in \boldsymbol{R}^{n},
$$

has a periodic orbit $\Gamma$ of period $T . \Gamma: \boldsymbol{x}=\gamma(t), 0 \leq t \leq T, \gamma(t+T)=\gamma(t)$. The linearization of Equation (8) about its periodic orbit $\Gamma$ is

$$
\delta \dot{\boldsymbol{x}}=A(t) \delta \boldsymbol{x},
$$

where $A(t)=\partial \mathbf{f}(\gamma(t)) / \partial \mathbf{x}$. A state transition matrix for Equation (9) is a nonsingular matrix $\Phi(t)$ which satisfies the matrix differential equation,

$$
\dot{\Phi}=A(t) \Phi
$$

For our system, the periodic matrix $A$ is

$$
A(t)=\left[\begin{array}{cccccc}
0 & 0 & 0 & 1 & 0 & 0 \\
0 & 0 & 0 & 0 & 1 & 0 \\
0 & 0 & 0 & 0 & 0 & 1 \\
U_{x x} & U_{x y} & U_{x z} & 0 & 2 \omega_{T} & 0 \\
U_{y x} & U_{y y} & U_{y z} & -2 \omega_{T} & 0 & 0 \\
U_{z x} & U_{z y} & U_{z z} & 0 & 0 & 0
\end{array}\right] .
$$

As we see the terms in matrix $A(t), U_{x z}=U_{y z}=U_{z x}=U_{z y}=0$ for planar motion $(z=0)$. This linear system can be decoupled by a linear transformation.

\section{SEARCHING FOR PERIODIC ORBITS}

Scheeres (2000) gave a general procedure to find periodic orbits near a given periodic orbit with a slightly different energy. Here we follow a similar idea, which reduces the state transition matrix (STM) from a $6 \times 6$ to a $4 \times 4$ matrix by removing the two unity eigenvalue associated with a periodic orbit in a time invariant system, then we can reduce our $4 \times 4$ STM $\Phi(T)$ into a $2 \times 2$ matrix $\Phi_{r r}(T)$. By giving a constraint to the initial value variation which keeps the Jacobi integral constant, we find an automatic approach to computing periodic orbits, which is an application of the Poincaré mapping. The Poincaré surface we used here is $y_{0}=0, \dot{y}_{0} \neq 0, y(T)=0$. The computation of these orbits requires a precision integration routine and a set of programs which allow one to force the end-points of an orbit to join up. 


\subsection{STM Dimension Reduction}

At the initial condition $y_{0}=0, \delta y_{0}=0$, and at the first return to the Poincaré surface $y(T)=0, \delta y(T+$ $\delta T)=0$. The system is expressed as

$$
\left[\begin{array}{l}
\delta x(T+\delta T) \\
\delta y(T+\delta T) \\
\delta \dot{x}(T+\delta T) \\
\delta \dot{y}(T+\delta T)
\end{array}\right]=\Phi(T)\left[\begin{array}{l}
\delta x_{0} \\
\delta y_{0} \\
\delta \dot{x}_{0} \\
\delta \dot{y}_{0}
\end{array}\right]+\left[\begin{array}{c}
\dot{x}(T) \\
\dot{y}(T) \\
\ddot{x}(T) \\
\ddot{y}(T)
\end{array}\right] \delta T
$$

The condition $\delta y(T+\delta T)=0$ allows us to solve the $\delta T$ necessary to stay on the Poincaré surface. Then we can reduce our system to

$$
\left[\begin{array}{l}
\delta x(T+\delta T) \\
\delta \dot{x}(T+\delta T) \\
\delta \dot{y}(T+\delta T)
\end{array}\right]=\left[\begin{array}{lll}
\phi_{r 11}(T) & \phi_{r 12}(T) & \phi_{r 13}(T) \\
\phi_{r 21}(T) & \phi_{r 22}(T) & \phi_{r 23}(T) \\
\phi_{r 31}(T) & \phi_{r 32}(T) & \phi_{r 33}(T)
\end{array}\right]\left[\begin{array}{l}
\delta x_{0} \\
\delta \dot{x}_{0} \\
\delta \dot{y}_{0}
\end{array}\right]
$$

where

$$
\phi_{r 11}(T)=\phi_{11}(T)-\frac{\dot{x}(T)}{\dot{y}(T)} \phi_{21}(T) \quad \ldots
$$

The Jacobi integral is restated as

$$
J=\frac{1}{2}\left(\dot{x}^{2}+\dot{y}^{2}+\dot{z}^{2}\right)-\frac{1}{2} \omega_{T}^{2}\left(x^{2}+y^{2}\right)-\frac{\mu}{r}-U_{2} .
$$

If we restrict $J$ to one value, then $\delta J=0$.

In the Poincaré surface $\delta y=0, \partial J / \partial \dot{y}=\dot{y} \neq 0$, allowing us to solve for $\delta \dot{y}$ as

$$
\delta \dot{y}=-\frac{1}{\frac{\partial J}{\partial \dot{y}}}\left(\frac{\partial J}{\partial x} \delta x+\frac{\partial J}{\partial \dot{x}} \delta \dot{x}\right)
$$

Evaluated at the initial conditions we can find the necessary variation in $\dot{y}_{0}$ to keep $J$ constant.

This allows a final reduction, equivalent to removing the two unity eigenvalues from the original $4 \times 4$ STM,

$$
\begin{aligned}
& {\left[\begin{array}{l}
\delta x(T+\delta T) \\
\delta \dot{x}(T+\delta T)
\end{array}\right]=\left[\begin{array}{ll}
\phi_{r r 11}(T) & \phi_{r r 12}(T) \\
\phi_{r r 21}(T) & \phi_{r r 22}(T)
\end{array}\right]\left[\begin{array}{l}
\delta x_{0} \\
\delta \dot{x}_{0}
\end{array}\right]} \\
& \equiv \Phi_{r r}(T)\left[\begin{array}{l}
\delta x_{0} \\
\delta \dot{x}_{0}
\end{array}\right]
\end{aligned}
$$

where

$$
\phi_{r r 11}(T)=\phi_{r 11}(T)-\frac{\partial J / \partial x_{0}}{\dot{y}_{0}} \phi_{r 13}(T) \quad \ldots
$$

For periodic orbits, there are some properties of the reduced order map $\Phi_{r r}(T)$. First, $\phi_{r 11} \phi_{r 22}-$ $\phi_{r 12} \phi_{r 21}=1$ due to volume conservation in phase space. Secondly, if our periodic orbit has $\dot{x}_{0}=0$, then $\phi_{r 11}=\phi_{r 22}$ due to symmetry of the orbit about the $x$-axis. A similar discussion can be found in Hénon (1973) for three body problem. 


\subsection{Search Procedure}

If the initial condition is $\left(x_{0}, \dot{x}_{0}\right)$, and its first return is $\left(x_{1}, \dot{x}_{1}\right)$, we wish to choose the correction $\delta x_{0}, \delta \dot{x}_{0}$, to drive

$$
\left[\begin{array}{c}
x_{1}+\delta x_{1} \\
\dot{x}_{1}+\delta \dot{x}_{1}
\end{array}\right] \rightarrow\left[\begin{array}{c}
x_{0}+\delta x_{0} \\
\dot{x}_{0}+\delta \dot{x}_{0}
\end{array}\right]
$$

To do this we solve for the correction that makes this occur for our linear system. Then the new initial condition can be written as

$$
\left[\begin{array}{c}
x_{0 n} \\
\dot{x}_{0 n}
\end{array}\right]=\left[\begin{array}{c}
x_{0} \\
\dot{x}_{0}
\end{array}\right]+\left[I-\Phi_{r r}(T)\right]^{-1}\left[\begin{array}{c}
x_{1}-x_{0} \\
\dot{x}_{1}-\dot{x}_{0}
\end{array}\right]
$$

By imposing the Jacobi integral invariant on the initial condition variation, using Equation (15), we have $\dot{y}_{0 n}$ by the following relationship when $x_{0}>0$,

$$
\dot{y}_{0 n}= \pm \sqrt{2\left(J-\frac{1}{2} \dot{x}_{0 n}^{2}+\frac{1}{2} \omega_{T}^{2} x_{0 n}^{2}+\frac{\mu}{x_{0 n}}-\frac{\mu}{2 x_{0 n}^{3}} C_{20}+\frac{3 \mu}{x_{0 n}^{3}} C_{22}\right)} .
$$

Thus, Equation (15) indicates

$$
J\left(x_{0 n}, y_{0 n}=0, \dot{x}_{0 n}, \dot{y}_{0 n}\right)=J\left(x_{0}, y_{0}=0, \dot{x}_{0}, \dot{y}_{0}\right) .
$$

There exist relations between the properties of the periodic orbit and the algorithm. If at the first return $\left(x_{1}, \dot{x}_{1}\right)$ is too far from $\left(x_{0}, \dot{x}_{0}\right)$, the searching algorithm diverges, and a periodic orbit does not exist near $\left(x_{0}, \dot{x}_{0}\right)$ for the given Jacobi integral. If $\operatorname{det}\left|I-\Phi_{r r}(T)\right| \approx 0$, the periodic orbit is at a critical point from stable to unstable, and at this point the searching program usually fails. We use these properties to modify our searching program by adding some limitations on the searching step size and recursive counting number, which makes our program more "automatic".

\section{STABILITY ANALYSIS}

Given a periodic orbit, we can use the Floquet Theorem to determine its stability by calculating its state transition matrix $\Phi(t)$ and its characteristic multipliers. If all its characteristic multipliers $e^{\lambda_{j} T} \leq 1$, then the periodic orbit is stable.

\subsection{Characteristic Multipliers}

For a periodic matrix $A(t)$, we have the following result known as Floquet's Theorem (see Perko 1991). If $A(t)$ is a continuous, $T$-periodic matrix, then for all $t \in \mathbf{R}$ any state transition matrix solution for Equation (9) can be written in the form $\Phi(t)=Q(t) e^{B t}$, where $Q(t)$ is a nonsingular, differentiable, $T$ periodic matrix and $B$ is a constant matrix. Furthermore, if $\Phi(0)=I$, then $Q(0)=I$. The eigenvalues of $e^{B T}$ are given by $e^{\lambda_{j} T}$ where $\lambda_{j}, j=1, \ldots, n$, are eigenvalues of the matrix $B$.

The eigenvalues of $B, \lambda_{j}$ are called characteristic exponents of periodic orbit $\gamma(t)$ and the eigenvalues of $e^{B T}, e^{\lambda_{j} T}$ are called characteristic multipliers of $\gamma(t)$.

It can be shown that one of the characteristic exponents of periodic orbit $\gamma(t)$ is always zero, i.e. one of the characteristic multipliers is always 1 , but for Hamiltonian systems there exist two unity multipliers since if $\eta$ is an eigenvalue of $\Phi(t)$ for a Hamiltonian system, its inverse $\eta^{-1}$, complex conjugate $\bar{\eta}$, and inverse complex conjugate $\bar{\eta}^{-1}$ are also its eigenvalues.

The product of all the characteristic multipliers is

$$
\prod_{i=1}^{6} e^{\lambda_{i} T}=e^{\left(\sum_{i=1}^{6} \lambda_{i}\right) T}=|\Phi(T)|=e^{\int_{0}^{T} \operatorname{tr}(A(q)) d q}=e^{0}=1 .
$$

Thus, two characteristic multipliers of the periodic orbit are unity, since the dynamics of the uniformly rotating problem is Hamiltonian.

At the orbit period $T, Q(T)=I, e^{B T}=\Phi(T)$ whose eigenvalues are the characteristic multiplies of the periodic orbits according to the Floquet's theorem. The calculated characteristic multipliers $e^{\lambda_{i} T}$ of the periodic orbits in Figure 1 are listed in Table 1. The two unity eigenvalues of the STM indicate the periodicity of the orbit, while the other two are just the index of stability of the periodic orbit. We see that only the near circular periodic orbits are stable, and all the other orbits are unstable. 


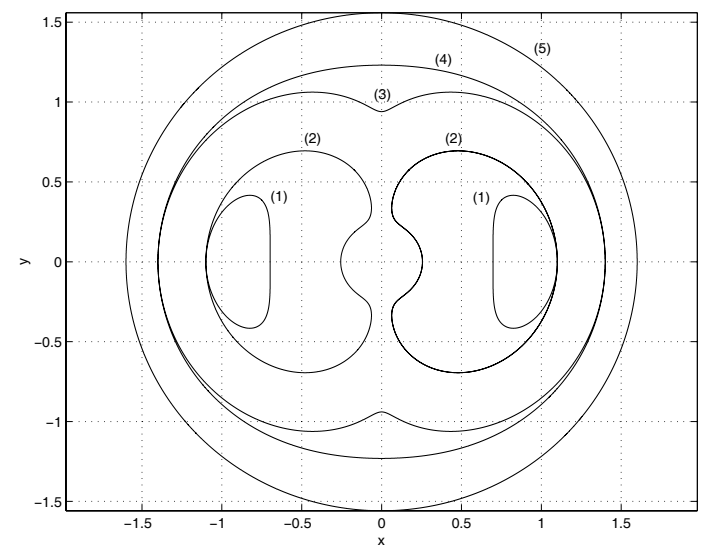

Fig. 1 Examples of periodic orbits when the initial value of $x_{0}$ ranging from 1.1 to $1.6, y_{0}=0$, and $\dot{x}_{0}=0$. For asteroid 4769 Castalia $\mu=9.40 \times 10^{-8} \mathrm{~km}^{3} \mathrm{~s}^{-2}, C_{20}=-7.275 \times 10^{-2} \mathrm{~km}^{2}, C_{22}=2.984 \times 10^{-2} \mathrm{~km}^{2}$, $\omega_{T}=4.2883 \times 10^{-4}$ radius s $^{-1}$. The four equilibria are $( \pm 0.9070,0)$ and $(0, \pm 0.7019)$. We note that the minimum radius of the lobe orbits are less than the dimension of the asteroid, and are shown for theoretical interest only.

Table 1 The Calculated Characteristic Multipliers $e^{\lambda_{i} T}$ of the Periodic Orbits in Fig. 1

\begin{tabular}{lccccc}
\hline orbit description & $x_{0}$ & $\lambda_{1}$ & $\lambda_{2}$ & $\lambda_{3}$ & $\lambda_{4}$ \\
\hline (1) small lobe & 1.1 & 90.50 & 0.0110 & 1.0 & 1.0 \\
(2) large lobe & 1.1 & 126.6 & 0.0079 & 1.0 & 1.0 \\
(3) double lobes & 1.4 & 1275 & 0.00078 & 1.0 & 1.0 \\
(4) near ellipse & 1.4 & 42.46 & 0.0236 & 1.0 & 1.0 \\
(5) near circle & 1.6 & $-0.846-0.533 \mathrm{i}$ & $-0.846+0.533 \mathrm{i}$ & 1.0 & 1.0 \\
\hline
\end{tabular}

\subsection{Characteristic Equation}

Since the system is Hamiltonian, the characteristic multipliers have a specific structure. The characteristic equation of the state transition matrix $\Phi(T)$ for planar orbits is

$$
\left(\eta^{2}-\left(k_{a}+1 / k_{a}\right) \eta+1\right)\left(\eta^{2}-2 k_{b} \eta+1\right)=0,
$$

where $e^{\lambda_{i} T}=\eta_{i}$, and $k_{a}$ and $k_{b}$ are two real numbers. Equation (24) describes the structure of the characteristic multiplies for our system, if $e^{\lambda_{1} T}=k_{a}, e^{-\lambda_{1} T}=1 / k_{a}, e^{\lambda_{2} T}=k_{b}+i \sqrt{1-k_{b}^{2}}$, $e^{\bar{\lambda}_{2} T}=k_{b}-i \sqrt{1-k_{b}^{2}},-1 \leq k_{a} \leq 1,-1 \leq k_{b} \leq 1$. For example, a stable near circular orbit, $k_{a}=1$, $\left|k_{b}\right|<1$ and an unstable lobe or elliptic orbit $\left|k_{a}\right|<1, k_{b}=1$. We note that for a real number, its complex conjugate is itself; and for a complex number on the unit-circle, its inverse is its conjugate.

Another way to analyze the stability of a periodic orbit is to calculate the coefficients of the characteristic equation without solving for the multipliers, and define a "stability index" which is similar to the variable $-\left(k_{a}+1 / k_{a}\right)$ or $-2 k_{b}$ in Equation (24). This method was presented in Szebehely (1967), where it was used for the restricted three body problem with two equal mass primaries. When $\left(k_{a}+1 / k_{a}\right)>2$, $k_{b}=1$ the periodic orbit is unstable; when $k_{a}+1 / k_{a}=2,\left|k_{b}\right|<1$ the periodic orbit is stable.

Considering the out of plane stability in planar motion, we can decompose the full six by six STMs into a $4 \times 4$ in-plane STM $\Phi$ and a $2 \times 2$ out-of-plane $\operatorname{STM} \Phi_{z}$.

Similarly we can have $\Phi_{z}(T)$ and its characteristic equation for a periodic orbit,

$$
\eta_{z}^{2}-2 k_{c} \eta_{z}+1=0 \text {. }
$$

Normally, $-1<k_{c}<1$, which means that the periodic orbit is stable in the out-of-plane direction, but some retrograde periodic orbits when their radii are close to the central body are in-plane stable and outof-plane unstable. This result is compatible with the secular motion analysis for a slowly rotating central 
body (Hu \& Scheeres 2002). The variable $k_{c}$ in Equation (25) corresponds to the "vertical stability index" in Hénon (1973), where he gave a discussion of the out-of-plane stability of periodic orbits in the restricted three body problem.

\section{FAMILIES OF PERIODIC ORBITS}

Figure 1 shows some periodic orbits, which are all direct orbits in inertial space, for asteroid 4769 Castalia. For retrograde orbits there always exist stable, near-circular periodic orbits, but for direct orbits the case is different. For example in our particular case, when $x_{0}=1.3$ and $x_{0}=1.4$ there will not be any near circular direct periodic orbits. This corresponds to the resonant region from 1:1 to 2:1 in Figure 5. When the orbit radius is large enough, say $x_{0} \geq 1.6$, direct near-circular periodic orbits exist as a continuous family along the $x$-axis. Additionally, at some radii, for example at $x_{0}=1.1$ and $x_{0}=1.4$, by changing the energy level, we can find more than one periodic orbit. Figure 2 shows examples of periodic orbits when the central body rotates slowly. When the central body rotates fast, some of these periodic orbits will disappear or change their stability character.

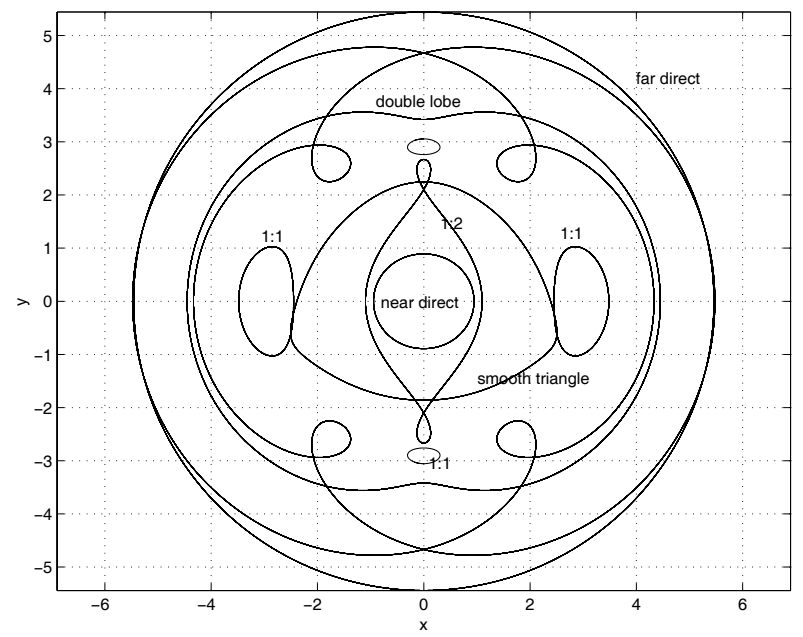

Fig. 2 Examples of periodic orbits when the central body rotates slowly, at $1 / 7$ th of the rotation rate of the asteroid Castalia. The four equilibrium points are $( \pm 2.9673,0)$ and $(0, \pm 2.9076)$.

\subsection{Equilibrium Points}

In essence, the equilibrium points are special kinds of periodic orbits of period $T=0$. By analyzing the eigenvalues $A_{r}$ at the equilibrium points, we can understand the stability and existence of the nearby periodic orbits. Similar to the results for an ellipsoidal central body, it can be shown that the equilibrium point along the $x$-axis always has an unstable saddle manifold and a center manifold. The equilibrium point along the $y$-axis is stable when the central body rotates slowly, and has two center manifolds. These points become unstable when the central body rotates fast enough, and then there will be one stable spiral manifold and one unstable spiral manifold.

The stability condition is (Hu \& Scheeres 2004)

$$
r_{\mathrm{s}}^{2}+C_{20}-162 C_{22}>0
$$

where the radius of a synchronous orbit when $C_{20}=C_{22}=0$ is $r_{\mathrm{s}}=\left(\mu / \omega_{T}^{2}\right)^{1 / 3}$.

\subsection{Classification}

The family of direct, equatorial, near circular and body-fixed periodic orbits have two branches, one near the asteroid (the small circle in Fig. 2), the other far from the asteroid (the large circle in Fig. 2). The near 
branch (with its $n \gg \omega_{T}$ ) disappears when the central body rotates fast. The far branch $\left(n \ll \omega_{T}\right.$ ) always exists and when the central body rotates fast it gets closer to the central body. The direct periodic orbits of both the near and far branches are usually stable when exist.

Between these two branches of direct periodic orbits are the 1:1 and 1:2 resonant periodic orbits. The 1:1 resonant orbits, whose mean motion rate $n=\omega_{T}$ (see Fig. 2), also have two branches. Their existence and stability have a close relationship with the equilibrium point analysis, as shown in Section 5.1.

One branch of the 1:1 resonant periodic orbit is around the equilibrium points along the short equatorial axis ( $y$-axis), which is present and stable when (see Equation (26))

$$
\omega_{T}<\mu^{1 / 2} /\left(-C_{20}+162 C_{22}\right)^{3 / 4} .
$$

The equilibrium point at the $y$-axis has two center manifolds, so nearby periodic orbits are present and stable. When the central body rotates faster, this equilibrium point has one stable and one unstable spiral manifold, then no periodic orbits exist near this equilibrium.

The other branch of the 1:1 periodic orbits is around the equilibrium point along the long equatorial axis ( $x$-axis), which is always present, but unstable. See Figure 1 for the fast rotation case. Since the equilibrium points along the $x$-axis have a center and a saddle manifold, periodic orbits exist near the equilibrium point but are unstable.

For the Earth, $C_{22}$ and $\omega_{T}$ are very small and the 1:1 resonant orbits can be very large, even encircling three of the four equilibriums, and their stability can also change. For example, in the family of 1:1 periodic orbits near the equilibrium point $\left(x_{\text {eq }}, 0\right)$, the smaller one is unstable, while the large one including the three equilibrium points $\left(x_{\text {eq }}, 0\right),\left(0, \pm y_{\text {eq }}\right)$ is stable (Lara 2000).

The near branch of direct periodic orbits meets the 1:2 resonant periodic orbit family and disappears as its radius increases. The 1:2 resonant orbit with mean motion $n=2 \omega_{T}$ is present and stable when the central body rotates slowly. The shape of the 1:2 resonant orbits can be seen in Figure 2, and it changes for different values of the Jacobi integral. The 1:2 resonant orbits also exist along the $y$-axis. Figure 2 just shows one $1: 2$ resonant orbit along the $x$-axis.

Retrograde, near circular periodic orbits in the equatorial plane move in the opposite sense of the asteroid rotation. In general, such retrograde orbits are stable and exist at almost all radii.

Between the above basic families of periodic orbits, there exist some special periodic orbits. For example, at the boundary between the 1:1 resonant and far direct periodic orbits there is a family of double lobe periodic orbits (Figs. 1 and 2). Their stability character also changes from stable to unstable as $\omega_{T}$ increases.

One special periodic orbit is seen in the middle of Figure 2 between the 1:1 and 1:2 resonant orbits: it is a smooth, triangular periodic orbit but is not symmetric about the $x$-axis, and by the initial assumption it is perpendicular to the $x$-axis. The result can serve as an example of how well our searching procedure works.

\subsection{Distribution}

Figure 3 shows the families of periodic orbit for a specific asteroid. The upper-left branch is the near-direct periodic orbits, continuing down are the 1:2 resonant orbits. The upper-right branch are the far-direct orbits. The bottom branch is the retrograde periodic orbits. In the middle are the 1:1 resonant, double lobe, smoothtriangle-like periodic orbits, etc. As the central body rotates faster, the whole curve will translate to the left, and the old left part of the curve will disappear.

Figure 4 shows another example of periodic orbits in a slowly rotating case. We find that the direct, 1:1 and 1:2 resonant periodic orbits also exist, but the critical boundary periodic orbits are different.

Figure 5 shows a critical line between stable and unstable near circular quasi-periodic orbits at different $\omega_{T}$ in inertial space and their relation to the mean resonant orbits when $\omega_{T} / n^{*}$ is a ratio of two integers. The stable orbits defined in the plots are the orbits whose semi-major axis and eccentricity are nearly constant on average, which means the energy and angular momentum of the orbit do not change on average. Their orientation may change, like the well known $J_{2}$ perturbation orbits around the Earth. If there are large changes in semi-major axis and eccentricity within an orbit, we regard this as unstable. We find in this picture when the line is crossed, the system is in a region with a more strongly varying semi-major axis and eccentricity. The near direct periodic orbit exists in the lower-left region where $\left(n-\omega_{T}\right) / \omega_{T} \gg 1$, which 
means $n \gg 2 \omega_{T}$, and the far direct periodic orbit exists in the upper-right region when $\left(\omega_{T}-n\right) / n \gg 1$, which means $\omega_{T} \gg 2 n$. The retrograde orbits exist when $\omega_{T}<0$. In the middle are the resonant lines, we see that the 1:2 resonant line acts like a thin wall which prevents the near direct periodic orbit from crossing it. We can prove that the near circular 1:2 periodic orbit is unstable in eccentricity. A 1:2 periodic orbit exists and is stable with relatively large eccentricity. We will publish these results based upon further analysis.

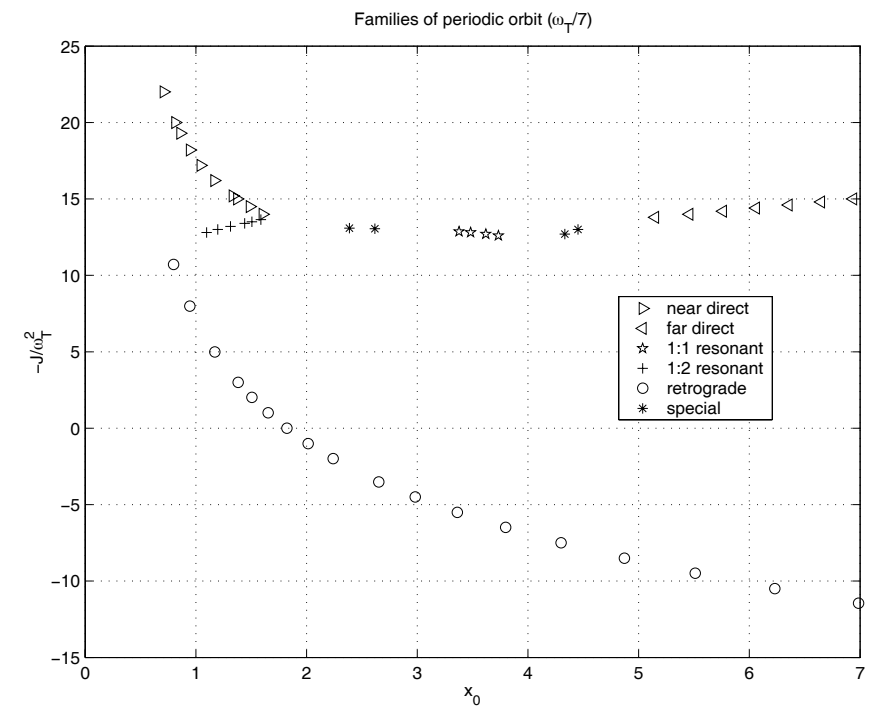

Fig. 3 Families of periodic orbit in the slowly rotating case corresponding to Fig. 2

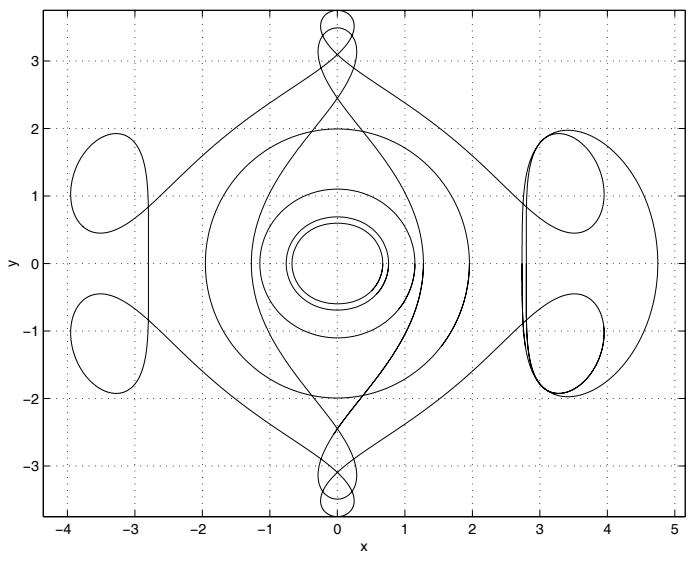

Fig. 4 Examples of periodic orbits at 1/10th of the rotation rate of asteroid Castalia.

For the near direct and some retrograde periodic orbits near the central body, when the central body rotates slowly, $\left(n-\omega_{T}\right) / \omega_{T} \gg 1$, secular perturbations make important contributions to the periodic motion (Hu \& Scheeres 2002).

\subsection{Summary}

We have found some basic families of periodic orbits around asteroids. The existence and stability of these families may change for different rotation rates. Table 2 lists the parameters of these periodic orbit families. 


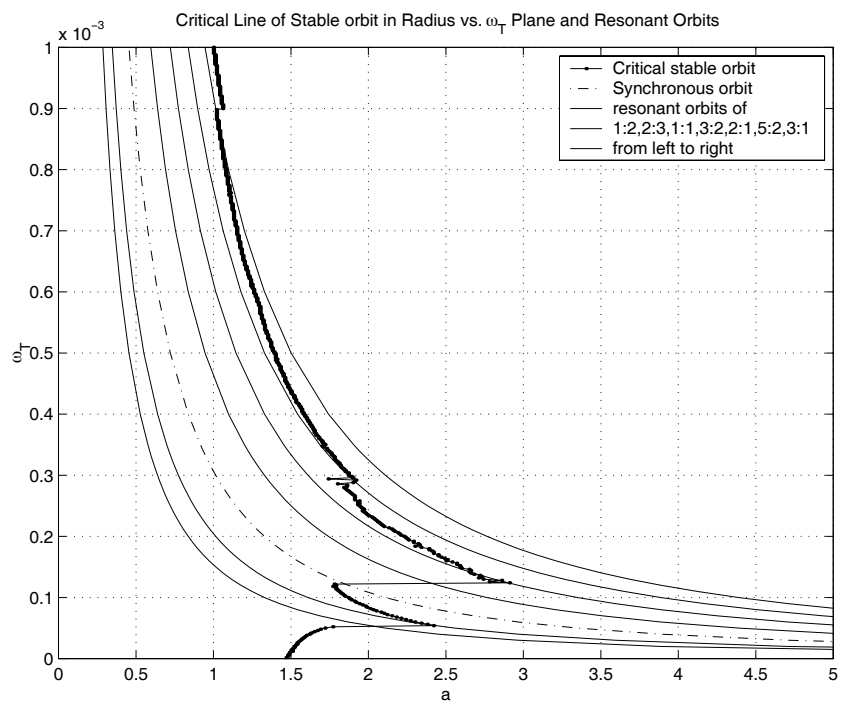

Fig. 5 Boundary line between "stable" and "unstable" regions of near circular, direct orbits in inertial space and their relation to resonant orbits when $\omega_{T} / n$ is a ratio of two integers. We find there are jumps at the resonances. The plot is made using the Castalia model with a varying rotation rate. For the actual asteroid, $\omega_{T}=4.2883 \times 10^{-4}$ radius $^{-1}$.

Table 2 A Summary of the Properties of Periodic Orbit Families

\begin{tabular}{lccc}
\hline periodic orbit & eccentricity & stability & existence \\
\hline near direct & $\mathrm{e} \approx 0$ & stable & $n \gg 2 \omega_{T}>0$ \\
far direct & $\mathrm{e} \approx 0$ & stable & $\omega_{T} \gg 2 n>0$ \\
$1: 1$ around $y_{\mathrm{eq}}$ & $\mathrm{e} \neq 0$ & stable & Equation $(27)$ \\
$1: 1$ around $x_{\mathrm{eq}}$ & $\mathrm{e} \neq 0$ & unstable & always \\
$1: 2$ resonant & $\mathrm{e} \neq 0$ & stable & $n \approx 2 \omega_{T}$ \\
retrograde & $\mathrm{e} \approx 0$ & stable & $n<0$ \\
special orbit & $\mathrm{e} \neq 0$ & stable/unstable & critical boundary \\
\hline
\end{tabular}

We note that some of the periodic orbits we discussed here have similarities to the periodic orbits of the restricted three body problem, and that all the periodic orbits checked, except the 1:2 resonant orbits, are the first return maps. There may be other periodic orbits which are second or third return maps similar to the three body problem as shown in Szebehely (1967).

\section{CONCLUSIONS AND DISCUSSION}

Periodic orbits in an arbitrary 2 nd degree and order uniformly rotating gravity field are studied. Equilibria can be regarded as periodic orbits with period zero. We see that the stability of these equilibria has a close relation with the existence and stability of their nearby periodic orbits. If the equilibrium manifolds have two centers, then nearby periodic orbits are present and stable; if the equilibrium manifolds are spiral, then periodic orbits do not exist; and if the equilibrium point has one center and one saddle manifold, then periodic orbits are present but unstable. By using a quasi-automatic searching procedure for periodic orbits, we have found five basic families of periodic orbits and checked their existence and stability at different central body rotation rates. There exist other periodic orbits, and they can be analyzed also by the above procedure. In this paper we focus on periodic orbits in planar motion. We find that retrograde periodic orbits are always stable and can be directly used as mission orbits. For applications of others periodic orbits, exquisite control should be taken with the long term evolution. 
Acknowledgements Helpful suggestions that largely improved the paper were given by Dr. Martin Lara, from Spain, when he visited the University of Michigan, and by an anonymous referee. This work was partially supported by an Innovation Fund from the Chinese Academy of Space Technology, and a grant from the Jet Propulsion Laboratory, which is under a contract with NASA.

\section{References}

Hu W., Scheeres D. J., 2002, AIAA J. of GCD, 25, 765

Hu W., Scheeres D. J., 2004, Planetary and Space Science, 52, 685

Kulkarni J. E., Campbel M. E., 2006, IEEE Trans. on Contr. Sys. Tech., 14, 572

Lara M., Elipe A., 2000, AAS/AIAA Space Flight Mech. Meeting, FL. USA

Macau E. N., 2003, Celest. Mech., 87, 3, 291

Perko L., 1991, Differential Equation and Dynamical System, New York: Springer-Verlag

Pravec P., Harris A. W., 2000, Icarus, 148, 12

Richardson D. L., 1980, AIAA J. of GCD, 3, 6, 543

Scheeres D. J., Ostro S. J., Hudson R. S. et al., 1996, Icarus, 121, 67

Scheeres D. J., Williams B. G., Miller J. K., 2000, AIAA J. of GCD, 23, 3, 466

Szebehely V., 1967, Theory of Orbits, Academic Press Inc., New York: Academic Press Inc. 